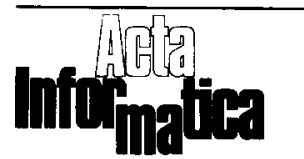

(C) Springer-Verlag 1981

\title{
Erratum
}

\section{General Purpose Schedulers for Database Systems}

\author{
M.A. Casanova and P.A. Bernstein \\ Acta Informatica 14, 195-220 (1980)
}

Definition 5.1 should read:

Definition 5.1. $(L, S) \in S E$ iff $L=\lambda \vee$

$$
\operatorname{elem}(L) \subset \operatorname{DOMAIN}(S) \wedge\left(\exists i_{1}, \ldots, i_{n} \in[1, \infty)\right)\left(L=R_{i_{1}} W_{i_{1}} \ldots R_{i_{n}} W_{i_{n}}\right) .
$$

Note that Definition 5.1 allows $i_{k}=i_{l}$, for some $k, l$. Hence, $(L, S) \in S E$ abstracts a serial computation of the transactions, possibly with repetitions. Moreover, note that weak serializability now has a slightly different interpretation.

With this change $\bar{H}_{D B S}$ becomes unnecessary, so that Lemma 5.1 now reads.

Lemma 5.1. Let $D B S=(V, A, n, S, S C D)$ be a database system and $H_{D B S}$ be a Herbrand interpretation for $D B S$.

$$
\bar{y} \in \bar{H}_{D B S} \quad \text { iff }(\exists(L, S) \in S E)\left(p_{H_{D B S}}[L, S](“ \bar{x} ”)=\bar{y}\right) .
$$

Theorem 5.2 remains the same, except that $\operatorname{Step}(5)$ is now unnecessary.

Proof of Lemma 5.1. $(\Leftrightarrow)$ Follows from the definition of $\bar{H}$ and $S E$.

$\Leftrightarrow)$ If $\bar{y}$ is a tuple of character strings, let $\lg (\bar{y})$ denote the sum of the lengths of all coordinates of $\bar{y}$.

Note that $\lg (\bar{y}) \geqq m$, if $\bar{y} \in \bar{H}_{D B S}$, where $m=\# V$. We prove the result by induction on $\lg (\bar{y})$.

basis: assume that $\lg (\bar{y})=m, \bar{y} \in \bar{H}_{D B S}$.

Then $\bar{y}=\bar{x}$. Since $p_{H_{D B S}}[\lambda, S](" \bar{x} ")=" \bar{x} "$, we are done.

Induction step: assume that the result holds for $\bar{y} \in \bar{H}_{D B S}$ such that $\lg (\bar{y})<k, k>m$. Let $\bar{y}^{\prime} \in \bar{H}_{D B S}$ be such that $\lg \left(\bar{y}^{\prime}\right)=k$. Since $\bar{y}^{\prime} \in \bar{H}_{D B S}$ and $k>m$, there must be $\bar{y} \in \bar{H}_{D B S}$ and $j \in[1, n]$ such that for any $i \in[1, m]$, if $x_{i} \notin S\left(W_{j}\right)$, then $y_{i}^{\prime}=y_{i}$ otherwise $y_{i}^{\prime}=$ " $f_{y i}\left(y_{j_{1}}, \ldots, y_{j_{i_{j}}}\right)$ ", where $S\left(R_{j}\right)=\left\{x_{j_{1}}, \ldots, x_{j_{t_{j}}}\right\}$. Then, $\lg (\bar{y})<\lg \left(\bar{y}^{\prime}\right)$. By the induction hypothesis, there is $(L, S) \in S E$ such that $p_{H_{D B}}[L, S](“ \bar{x}$ ") $=\bar{y}$. Construct now $L^{\prime}=L R_{j} W_{j}$. Then, we have that

$$
p_{H_{D B S}}\left[L^{\prime}, S\right](“ \bar{x} ")=p_{H_{D B S}}\left[R_{j} W_{j}, S\right]\left(p_{H_{D B S}}[L, S](“ \bar{x} ”)\right)=p_{H_{D B S}}\left[R_{j} W_{j}, S\right](\bar{y})=\bar{y}^{\prime}
$$

This concludes the proof.

Acknowledgment: We thank Prof. D.J. Rosenkrantz for pointing out a counter-example to the previous formulation of Lemma 5.1 . 